\title{
Design of encrypted transceiver non-coherent OFDM with ability to correct coding bits of information
}

\author{
Kareem J. Thajeel ${ }^{1}$, Izz K. Abboud ${ }^{2}$, Laith A. Kunbar ${ }^{3}$ \\ ${ }^{1}$ Baghdad Institution of Technology, Middle Technical University, Iraq \\ ${ }^{2,3}$ Computer Engineering Department, Al-mustansiriyah University, Iraq
}

\begin{tabular}{l} 
Article Info \\
\hline Article history: \\
Received Jan 21, 2019 \\
Revised Mar 15, 2019 \\
Accepted Apr 3, 2019
\end{tabular}

Keywords:

DQPSK

LDPC

SISO-OFDM

TSFE

\begin{abstract}
A differential encrypted transceiver was designed in the signal-to-noise ratio systems. A time-spread frequency-encoded (TSFE) algorithm was proposed using the orthogonal frequency multiplexing multiplier OFDM. The proposed design was verified under the Doppler frequency effect and there was an improvement in the performance of the signal-to-noise ratio system. As a result, the reliability of decoded data was increased and achieves the ability to correct coding bits of information.
\end{abstract} All rights reserved.

Corresponding Author:

Kareem J. Thajeel,

Baghdad Institution of Technology,

Middle Technical University,

Baghdad, Iraq.

Email: kareemjabbar847@yahoo.com

\section{INTRODUCTION}

Over the years, blind detection of orthodontic frequency division (OFDM) signals for selective frequency channels has been of great interest. The OFDM modulation fights the effect of multiple-path fading by channeling selective channels of frequencies to channels parallel to flat fading. Given that the OFDM sub-carrier carriers are closely related, the differential encoder along the sub-carrier lines of the code itself served. For a moderate consistency time, the sub-carriers of the successive OFDM codes have a strong relationship, making them suitable for differential coding over time. Thus, OFDM modulation permits DE concerning statistics bits over each frequency and time [1,2].

DE presents applicable deflect so pilot-assisted trough assessment is not potential because fast fading [3]. In addition, little signal-to-noise ratio (SNR) systems, achievement aqueduct canal status intimation (CSI) have a turn over effect of bandwidth competence. In such a scenario, DE together with duplication coding making use of inadvisable discovery affords because of and deflects solution. Note to that amount non coherent (dd) differential detection suffers beyond $3 \mathrm{~dB}$ SNR loss which in contrast according for exoteric disclosure [4].

High intricacy common differential detection for varied data codes is offered to get better performance [5, 6]. In Wi-Fi communication, order after decrease Bit Error Rate (BERs), there are two points affect, ability to acquire LDPC codes and, faster codes $[7,8]$. In $[9,10]$ that have been analyzing the implementations for LDPC blades yet Turbo blades through the use of Rayleigh fading channels. FDMimplication conflicts the fading over a multi-track trough yet permits little-intricacy receiver design. (DE) via hesitancy eclectic channels using OFDM description is suggested in [11, 12]. 
OFDM modulation authorizes DE over epoch or frequency dimension beneath younger yet moderate alacrity offering each era or frequency varieties. In DE alongside hesitancy then day orientations of OFDM marking, indicated with the aid of hesitancy-field differential modulation or time-domain differential modulation, be scrupulous. Standard differential revelation bears of a BER error-floor beneath distinctive channel situations. Problem about bad overall execution be handled in [13] utilizing semi-blind revelation in expenditure for leader tokens. The OFDM modulation authorizes DE on era or frequency distances under little or mild alacrity width both age yet frequency varieties. The traditional differential detection suffers from a BER error-floor beneath different channel. The trouble on bad execution is handled in [14] utilizing semi-blind discovery in consumption for leader tokens. Suggested TSFE style collectively together with DE, which addresses the BER error-floor dead end, then obtains higher variety through multipath Rayleigh fading channels (RFC) at the despicable viable virtue calculation. In [15-17] accomplishment about encoded DE upstairs day then frequency sizes are likened in the entity regarding Doppler frequency. High-intricacy common differential decoding for varied data codes is considered in $[18,19]$. This employment agree a DE steep transceiver styling as obtains short intricacy, younger vigor, and thriving range concerning single-input or single-output OFDM (SISO-OFDM) marking. Cautioned transceiver invests into little SNR systems in non-coherent method underneath multi-route canals besides channel state information.

Achieving total duct variety by periodical little-vigor DE records tokens atop subcarriers yet spreading over period. TSFE mode, labor tasks the reality as sequential subcarriers bear a dynamic feeling in accordance with usage DE upon subcarriers. It attains aggregation duct range via spreading differentiallyencoded information upon day and appropriating collections regarding uncorrelated subcarriers in conformity with differentially-encoded tokens indicated with the aid of superior interleaving. Examining BER accomplishment for recommended anger beneath Doppler frequency.

Performance comparisons were also made between optimization and randomization of sub-carriers in the relative mobility of nodes. To further improve the reliability of proposed modulation schemes, also the ability to code LDPC FEC was integrated with the design of the proposed transceiver. Simulation test explain up to expectation DE along TSFE be bendy after Doppler frequency then achieve higher BER of SNR.

\section{STATEMENT OF PROBLEM AND SOLUTION}

In telecommunications, orthogonal frequency-division multiplexing (OFDM) is a technique for encoding digital data on multiple carrier frequencies. OFDM has formed into a well-known plan for wideband digital communication, utilized in applications, for example, advanced TV and sound telecom, DSL web access, wireless networks, control line systems, and 4G portable interchanges. The primary issue in this article is the signal to noise ratio in SISO-OFDM with LDPC decoder must be limit, along these lines a time spread frequency-encoded (TSFE) algorithm was proposed utilizing the orthogonal frequency multiplexing multiplier (OFDM). The proposed plan was checked under the Doppler frequency effect and there was an enhancement in the execution of the signal-to-noise ratio system.

\section{BLOCK DIAGRAM OF TRANSCIEVER}

The SISO-OFDM signing in little SNR systems through Rayleigh fading channel for important routes $\mathrm{P}$, accordingly shown in Figure 1 . The cause of deeming a little SNR system arises of the fact that beneath deep fading, a transmitted indicative exposed to sharp canal attenuation, out coming in little SNR. At those scripts, earning from CSI is not applicable. Blind detection of a DE indicative supplies a substitution resolution to coherent detection. Spreading (recurrence) of DE input tokens over frequency or time sizes of an OFDM method conflicts intense channel weaklings to attain the aim energy per bit to noise ratio.

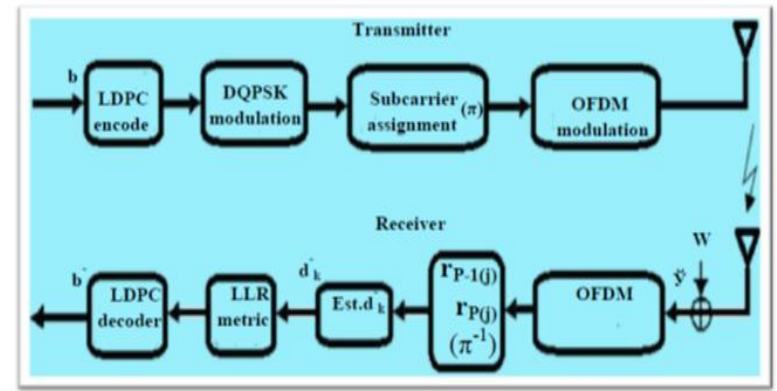

Figure 1. Block diagram of SISO-OFDM with LDPC decoder 
Moreover, even in mild and little channel debility, a transmitter sends by little power to attain a little prospect of disclosure. Like script, aim ability per bit to noise ratio be likewise accomplished through considering input pervasion over hesitancy sizes about an OFDM modulation.

\subsection{The transmitter}

According to the blocking graph namely shown in Figure 1, the LDPC encoder because of articles quantity $R=1 / 2$ encrypts enter piece wheel $b$ on $q$ pieces for a code phrase c concerning $n$ pieces. DE below a Gray planning plans code word $\mathrm{c}$ in imitation of a complicated constellation put in the number of elements in the group M. DE plans statistics bits in accordance with segment changing among the complicated constellation pots inherited through $(\ell-1)$ th or $\ell$ th subcarriers for same OFDM token. Traditional DE encrypts input along time [20]. It is noted in the situation of D $\backslash \mathrm{E}$ over OFDM subcarriers, input bits be encrypted for phase variation among input tokens inherited through sequential subcarriers from OFDM token. Block diagram in Figure 1 likewise deems proportional move from the connection contract. Attain aim ability every bit for noise proportion, suggest mode propagation DE input tokens over a time size. Allow $\Gamma$ be

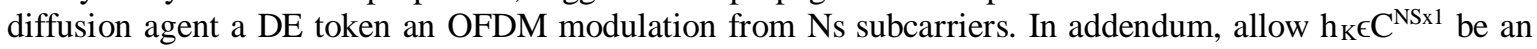
anonymous earning wheel from Ns subcarriers for OFDM order, $\mathrm{K}$ be time indicator. Anonymous earning wheel $h_{K}$ be at actuality a fast Fourier transformation (FFT) from casual channel vector $\tilde{h}_{\mathrm{K}}$ among sender and recipient, couple for zero-mean and difference $\sigma_{h}{ }^{2}=1 / \mathrm{P}$. which $\mathrm{h}_{\mathrm{k}}=\mathrm{Fh}_{\mathrm{k}}{ }_{\mathrm{k}}$ where $\mathrm{F}$ be a FFT mold. For DE input over subcarriers of OFDM regime, as we knew the truth that couple of sequential subcarriers for an OFDM regime has sturdy liaison, this liaison among sequential subcarriers raises with raising the number for OFDM subcarriers (Ns), then attain aim power per bit to noise ratio via pervasion DE input tokens over period. To attain utmost variety, subcarriers transferred to encrypted tokens must be minimal from connection; that is, equalize figure of separate channel routes, $\mathrm{P}$, of a hesitancy eclectic channel. $\Gamma$ is pervasion agent along time for OFDM token, (Ns - 1) data tokens be sender through $\Gamma$ OFDM tokens utilizing $\Gamma$ interleavers, $\pi_{1}, \pi_{2} \ldots \pi_{\Gamma}$. Looking DE over subcarriers is solid at proportional mobility for communicating nodes. That is because the use of TSFE method which dealing with time and frequency.

Figure 1 explain total system sample. Contemplate a binary writing series $\mathrm{L}=\left[\mathrm{L}_{1} \mathrm{~L}_{2} \ldots \mathrm{L}_{\mathrm{k}}\right]$, wherever every bit $\mathrm{L}_{\mathrm{k}}$ It is based on value zero or one for egalitarian chances, with regard to $\mathrm{k}=1,2 \ldots$. Letter succession be encrypted via average $\mathrm{K} / \mathrm{N}$ encrypting at word used for secrecy $\mathrm{W}=\left[\mathrm{W}_{1, \mathrm{MSB}} \mathrm{W}_{1, \mathrm{LSB}}\right.$ $\mathrm{W}_{2, \mathrm{MSB}} \mathrm{W}_{2, \mathrm{LSB}} \mathrm{W}_{3, \mathrm{MSB}} \mathrm{W}_{3, \mathrm{LSB}} \ldots$. .], wherever $\mathrm{N}$ represents positive integers. Wherever $\mathrm{N}$ represents positive integers. Utilizing QDPSK send off so expressed as in equation below (the signal goes to the target) $\mathrm{g}=[\mathrm{g}(0)$ $, \mathrm{g}(1), \mathrm{g}(3)$. . ], complicated information sign sent in period immediate $p$ be $\sim \mathrm{g}(p)=\mathrm{e}_{s}^{1 / 2} e^{\mathrm{j} \psi(p)}$, with regard to $p=0,1,3 \ldots$, wherever es , $\psi(p)$ be power, and stage for sign. At first, $\psi(0)$ laws like a reference so don't consists of no information in code word. At any sending period, information for twain blade pieces, $\mathrm{W}_{P,}$ MSB as well $\mathrm{W}_{P, \mathrm{LSB}}$, be jointly for phase variation for twain consecutive sign $\sim \mathrm{g}(p), \tilde{\mathrm{g}}(p-1), \ldots$ [21].

$$
\begin{aligned}
& \psi(p)=\psi(p-1)+\Delta \psi(p) \\
& \Delta \psi(p)=\left\{\begin{array}{c}
45 \text { whether } W_{P, M S B}=0, W_{P, L S B}=0 \\
135 \text { whether } W_{P, M S B}=0, W_{P, L S B}=1 \\
-45 \text { whether } W_{P, M S B}=1, W_{P, L S B}=0 \\
-135 \text { whether } W_{P, M S B}=1, W_{P, L S B}=1
\end{array} \quad \text { for } k=1,2,3, \ldots\right.
\end{aligned}
$$

The signal concatenation is sent for strange transporter phase. The extradited signal $(\tilde{E})$ is,

$$
\tilde{E}=\tilde{E}(0) * \tilde{E}(1) * \tilde{E}(2)
$$

which can follow similarly to,

$$
\tilde{E}(P)=\tilde{E}(P) e^{j \psi}+\tilde{n}(P), p=0,1,2
$$

Wherever $\psi$ indicates obscure transporter phase which statistical modeling be performed like random changing, on one form doling out during period $[-\pi, \pi)$. Stir (noise) concatenation $\{\sim \mathrm{s}(p)\}$ be random Gaussian changing for independence as well a single distribution for $\mathrm{D}[\sim \mathrm{s}(P)]=0$, D $\left[\left.\right|^{\sim} \mathrm{s}\left(\left.P\right|^{2}\right]=\mathrm{N}_{0}\right.$. In recipient, metrical for every symbol bit is calculated utilizing metrical calculator. These worth's be gone by into repeated LDPC encrypted wherever evaluated letter sequence ${ }^{\wedge} \mathrm{L}$ be gained like output. DE plans datum bits at phase move amidst complicated constellation dots referred through $(\ell-1)$ th, $\ell$ th subcarriers for selfsame OFDM token. Traditional DE encodes datum over time [22]. The datum bits in the phase difference be encrypted amidst the datum tokens sent over the sequential subcarriers for same OFDM token. 
The regulation sample in Figure 1 so looks proportional mobility for communicating nodes. To realize an aim suggested manner propagation DE datum tokens over a time distance. Allow $\Gamma$ exist the pervasion agent for DE token for OFDM modulation for Ns subcarriers. In a TSFE mode, input be DE over subcarriers and propagation over time. To DE input over subcarriers, relying in truth that adjoining subcarriers for OFDM regulation have altitude liaison, as offering at Figure 2. Correlation factor, $r_{P}(v)$, through 2 sequential subcarriers $(\mathrm{v}=1), \mathrm{h}_{\mathrm{P}}(\ell)$ and $\mathrm{h}_{\mathrm{P}}(\ell+1)$, raises through rising numeral for subcarriers $(\mathrm{Ns})$ of particular channel; $\mathrm{p}$ be period indicator. Observe which subcarriers conforming for little earning hardly deform encoded information. Conflicting the nulling impact for OFDM subcarriers via interleaved propagation for DE input tokens over period. OFDM modulation for Ns subcarriers DEs (Ns - 1) input tokens utilizing first subcarrier like a reference token. Because of big numeral for subcarriers, overhead because of reference subcarriers be humble. Repetition for encoded input tokens along freelance subcarriers fulfills aim power every bit to noise proportion and elevated variety in same time.

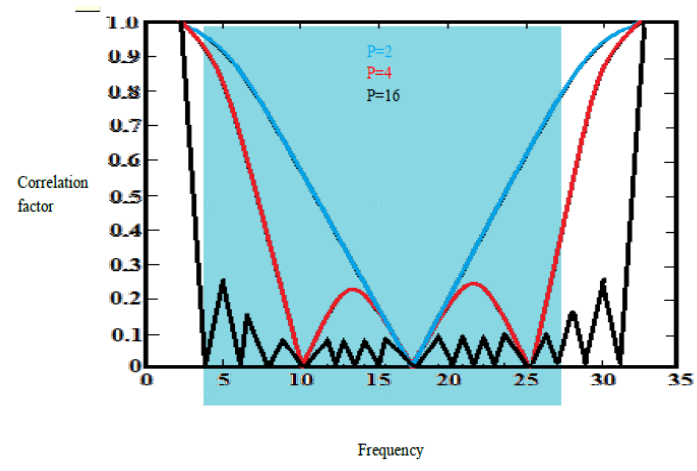

Figure 2. Auto-correlation of OFDM subcarriers

TSFE mode deeds frequency and time variety through using $\Gamma$ interleavers 1 with every recurrence for frequency encrypted OFDM token. Interleaver $\pi_{\mathrm{L}}$, wherever $\mathrm{L}=1 \ldots \Gamma$ appoints subcarriers to $\mathrm{DE}$ for every phase to $\mathrm{L}_{\mathrm{th}}$ OFDM token. Let $\Delta \psi=\left[\Delta \psi_{1} \Delta \psi_{2} \Delta \psi_{3 \ldots} \Delta \psi_{\mathrm{Ns}-2} \Delta \psi_{\mathrm{Ns}-1}\right]^{\mathrm{T}}$.

Indicate phase variances through tokens transmitted during Ns sequential subcarriers, performing of mapping for input bits for phase variances through tokens transmitted during sequential subcarriers to OFDM regime. Build $\Gamma$ DE token vectors for longitude Ns, every from interleaving phase variances wheel $\Delta \psi \ldots$

Stage variance vector $\Delta \psi \mathrm{L}=\pi_{\mathrm{L}}(\Delta \psi \mathrm{L})$, wherever $\mathrm{L}=1 \ldots \Gamma$. Knowing $\Delta \psi_{1}=\pi_{1}(\Delta \psi)=\Delta \psi . \mathrm{DE}$ token model for TSFE style conforming for $\Gamma$ stage variance vectors broadcast through $\Gamma$ OFDM tokens be

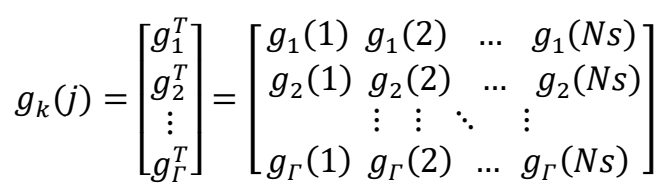

As seen [1], $g_{k}$ (j) represents data token Going with a signal destined towards the target on jth subcarrier for Lth OFDM token. Allow $d(j)=[d 1(j), d 2(j), \ldots, d \Gamma(j)]^{T}$ be brink signals for the sub wave that carries the information to stage variance $\Delta \psi 1$ at wheel $\Delta \psi$, wherever $\mathrm{j}=1, \ldots$, Ns -1 .

$$
\Delta \Psi_{1}=\left\llcorner\left\{ S^{*} L[d l(j)+S L[d L(j)]\}\right.\right.
$$

Every token SL (dL (j)) be coated with Gain, from the OFDM sub-wave bearing the signal, $\mathrm{hL}(\mathrm{dL}(\mathrm{j}))$ knowing the 1 st token for every OFDM token, $g_{k}(1)$, represent a reference indicative for DE. Whether receiver include information for canal arrange, $\mathrm{P}$, thereafter its ability assign sub-wave bearing the signal for DE data token succession utilizing a perfect interleaver for realize total canal variety. The mission for sub-wave bearing the signal does not variation if the same canal command remains.

Perfect interleaving to recognized canal command $\mathrm{P}$, linkage function amidst sub-wave bearing the signal for OFDM inclusion be $[23,24]$. In the following statements interleaving will now be clarified:

$$
\left.r_{p}(v)=E H_{p}^{*}(\ell+V)\right)=\sum_{n=0}^{P-1} \sigma_{h}^{2} e^{\frac{i 2 \pi v n}{N s}}
$$

Wherever $v$ be indicator for linkage during subcarrier earns $h_{k}(\ell)$ as well $h_{k}(\ell+v)$. 
Variety arrangement $\mathrm{P}$, for Multi-track canal, could fulfill via gathering OFDM subcarriers at sets from disconnected subcarriers. Figure 2 shows linkage factors for OFDM subcarriers of Ns equal thirty-two to channel commands $\mathrm{P}$ equal two, four, and sixteen. Linkage factors be 0 of $\mathrm{D}, 2 \mathrm{D} \ldots(\mathrm{P}-1) \mathrm{D}$, wherever $\mathrm{D}=\mathrm{Ns} / \mathrm{P}$. In generality $\Gamma$ equal $\mathrm{P}$ elements for 0 linkages that could earn total variety $\mathrm{P}$. So, for earn total variety, the circulation coefficient $\Gamma$, must be maximal than or equal for P. It assigns 1 set of uncorrelated subcarriers for a DE input token to earn total variety. For $\Gamma$ maximal $\mathrm{P}$, then minimum $\mathrm{P}$ disconnected subcarriers. For TSFE program, $1 \mathrm{DE}$ input token is sending at $\Gamma$ sequential OFDM tokens along uncorrelated subcarriers. As model, if having $\mathrm{P}$ equal four and Ns equal thirty-two subcarriers, so it consists of 4 disconnected channel earns at set; $\Gamma=\mathrm{P}=4$. So, 1 phase variance, $\Delta \psi \mathrm{j}$, could send along brinks at 4 uncorrelated subcarriers to $\Gamma=4$. Moreover, accurate for optimal interleaving, allow gaze model wherever Tequal four, Pequal four, and Ns equal sixteen. So, it consists of 4 optimal interleavers which propagation a phase variance vector for magnitude fifteen identical for DE at 4 sequential, OFDM tokens. So, the demand 4 optimal interleaves be shown below: interleaves be shown below:

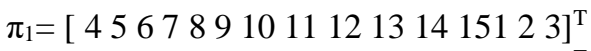

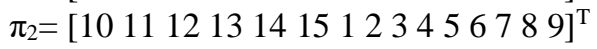

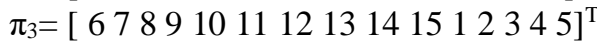

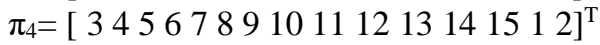

In relation to the previous model, it demands a phase variance of $\Delta \psi_{1}=L\left(\mathrm{~s}_{1} *(2) \mathrm{s}_{1} *(2)\right)$ of OFDM token, Depending on the interleaver $\pi_{1}$. The phase variance $\Delta \psi_{1}$ be propagation along 4 sequential OFDM tokens. Similarly, with regard to $\pi_{2}, \pi_{3}$, as well $\pi_{4}, \Delta \psi_{1}=\left\llcorner\left(\mathrm{g}_{2} *(6) \mathrm{g}_{1} *(5)\right),\left\llcorner\left(\mathrm{g}_{3} *(10) \mathrm{g}_{3} *(9)\right)\right.\right.$, and $L\left(g_{4} *(14) g_{4} *(13)\right)$. Knowing gaining perfect interleaver $\pi \mathrm{L}$ to $L$ th OFDM token via circularly variable interleaver $\pi \mathrm{L}-1$ with $\mathrm{D}$ equal $\mathrm{Ns} / \Gamma$, wherever $\mathrm{L}$ equal $1 \ldots \Gamma$.

Extension coefficient $\Gamma$ fulfills variety $\mathrm{P}$ via propagation $\Delta \psi$ along $\Gamma \geq \mathrm{P}$ OFDM tokens and through appoints uncorrelated subcarriers for encoded input tokens. Channel arrangement could evaluate without experiment assist utilizing standing modes [25, 26]. If the channel command be unknown or channel cohesion time be little, optimal customization for subcarriers isn't likely and random subcarrier Customization be applicable solution.

\subsection{The receiver designs}

The DE model resists channel weaklings and spectrum invalids for OFDM subcarriers through propagation encoded input over time at $\Gamma$ OFDM tokens. Allow $\breve{\mathrm{Y}}_{\mathrm{L}}$ be the monitoring vector conformable for kth OFDM token. next take off the cyclic prefix $(C P)$ of $\breve{y}_{L} \in c^{(N s=p) x}$, it has $\breve{y}_{L}(\ell)$ such demodulated monitoring conformable for $\ell$ th sub-wave bearing the signal, wherever $\ell=1, \ldots$, Ns and $\mathrm{y}_{\mathrm{L}}=\mathrm{FFT}\left(\breve{Y}_{\mathrm{L}}\right)$ $\epsilon \mathrm{C}^{(\mathrm{Ns}=\mathrm{p}) \mathrm{x}}{ }_{1}$.

Phase variance $\Delta \psi$ between 2 tokens over subcarriers, that appears bit input be propagation over time of TSFE model at $\Gamma$ epochs. The vector $y_{L}$ appears monitoring conformable for (Ns- 1) phase variances encrypted over subcarriers, and $\mathrm{L}$ appears period indicator. Time indicator be restarting to one next $\Gamma$ OFDM tokens. Which be, $\mathrm{L}=1 \ldots \Gamma$, wherever $\mathrm{L}$ be indicator for OFDM token conformable for propagation for hesitancy encoded input token vector $\Delta \psi$. phase variance vector sender along Lth OFDM token be $\Delta \psi_{\mathrm{L}}=\pi_{\mathrm{L}}$ $(\Delta \psi)$. As is known $\mathrm{d}_{1}(\mathrm{j}), \mathrm{d}_{2}(\mathrm{j}) \ldots \mathrm{d}_{\Gamma}(\mathrm{j})$ be brink index for subcarriers assigned for phase variance $\Delta \psi_{\mathrm{j}}$ at wheel $\Delta \psi$ wherever $\mathrm{j}=1 \ldots$ Ns- 1 . So

$$
\mathrm{r}_{1}(\mathrm{j})=\left[\begin{array}{c}
y 1(\operatorname{dj}(1)) \\
\vdots \\
y \Gamma(\operatorname{dj}(\Gamma))
\end{array}\right]=\left[\begin{array}{c}
h 1(\operatorname{dj}(1)) s(\operatorname{dj}(1)) \\
\vdots \\
h \Gamma(\operatorname{dj}(\Gamma)) s(\operatorname{dj}(\Gamma))
\end{array}\right]+\left[\begin{array}{c}
W 1(\operatorname{dj}(1)) \\
\vdots \\
W \Gamma(\operatorname{dj}(\Gamma))
\end{array}\right]
$$

and

$$
\mathrm{r}_{2}(\mathrm{j})=\left[\begin{array}{c}
h 1(\operatorname{dj}(1)) s(\operatorname{dj}(1)+1) \\
\vdots \\
h \Gamma(\operatorname{dj}(\Gamma)) s(\operatorname{dj}(\Gamma)+1)
\end{array}\right]+\left[\begin{array}{c}
W 1(\operatorname{dj}(1)+1) \\
\vdots \\
W \Gamma(\operatorname{dj}(\Gamma)+1)
\end{array}\right]
$$

With Note $\Delta \psi_{\mathrm{j}}=\left\llcorner\left(\mathrm{s}^{*}(\mathrm{dj}(1)+1) \mathrm{s}\left(\mathrm{d}_{\mathrm{j}}(1)\right)=\mathrm{e}^{-\mathrm{j} \Delta \psi_{\mathrm{j}}}\right.\right.$ dispreading be appeared as below:

$$
d^{\wedge}(j)=r_{1}^{H}(j) r_{2}(j), \Delta \psi_{j}^{\wedge}=L d^{\wedge}(j)
$$


wherever $\mathrm{H}$ indicates the Hermitian conjugate for vector. So, evaluation of $(\mathrm{Ns}-1)$ elements from vector $\Delta \varphi$ be estimated through building (Ns -1$)$ couple for $r_{1}(j)$ and $r_{2}(j)$ wheels as well via utilizing $(9)$. Using $d^{\wedge}(j)$ till assessment $\Delta \psi_{\mathrm{j}}$ as well LLR; so, its ha

$$
\begin{aligned}
& d^{n}(j)=r_{1}^{H}(j) r_{2}(j) \\
& \quad=\sum_{m=1}^{\Gamma}\left|h_{L}(d j(L))\right|^{2}|s(d j(L))|^{2} e^{i \Delta^{\phi}}{ }_{j} \\
& \quad=d^{n}(j)+W_{e(j)}
\end{aligned}
$$

wherever

$$
\begin{aligned}
W_{e(j)}= & h_{1}^{*}\left(d_{j}(1)\right) s^{*}\left(d_{j}(1)\right) W_{1}\left(d_{j}(1)+1\right) \\
& +h_{1}\left(d_{j}(1)\right) s\left(d_{j}(1)+1\right) W_{1}^{*}\left(d_{j}(1)\right) \\
& +W_{1}^{*}\left(d_{j}(1)\right) W_{1}\left(d_{j}(1)+1\right) \\
& +h_{2}^{*}\left(d_{j}(2)\right) s^{*}\left(d_{j}(2)\right) W_{1}\left(d_{j}(2)+1\right) \\
& +h_{2}(d(2)) s\left(d_{j}(2)+1\right) W_{2}^{*}\left(d_{j}(2)\right) \\
& +W_{2}^{*}\left(d_{j}(2)\right) W_{2}\left(d_{j}(2)+1\right) \\
& +h_{\Gamma}^{*}\left(d_{j}(\Gamma)\right) s^{*}\left(d_{j}(\Gamma)\right) W_{\Gamma}\left(d_{j}(\Gamma)+1\right) . \\
& +h_{\Gamma}\left(d_{j}(\Gamma)\right) s\left(d_{j}(\Gamma)+1\right) W_{\beta}^{*}\left(d_{j}(\Gamma)\right) \\
& +W_{\Gamma}^{*}\left(d_{j}(\Gamma)\right) W_{\Gamma}\left(d_{j}(\Gamma)+1\right), \\
& W_{e}(j)=\sum_{L=1}^{\Gamma}\left[h _ { L } ^ { * } \left(d j(L) S^{*}(d j(L)) W_{\Gamma}(d j(m L)+1]\right.\right. \\
& +h_{L}\left(d_{j}(L)\right) s\left(d_{j}(L)+1\right) W_{L}^{*}(d(L)) \\
& +W_{L}^{*}\left(d_{j}(L)\right) W_{L}\left(d_{j}(L)+1\right)
\end{aligned}
$$

Knowing sub-wave bearing the signal canal accomplishment $h_{p}(j)$, stir accomplishment $W_{p}(j)$, and encrypted tokens $S_{p}(j)$ be separate for 0 -mean. Thus, difference $\sigma^{2} \mathrm{e}$ for active stir

$$
W_{e}(j) b e \sigma^{2} e=2 \Gamma^{2} N_{0}
$$

wherever $\mathrm{N}_{0}$ be difference for additive white Gaussian noise. The evaluation for stage variance vector $\Delta \psi$ of (Ns - 1) stage variances for TSFE style be

$$
\Delta \psi_{k}^{\wedge}=\left[\Delta \psi_{1}^{\wedge} \Delta \psi_{2}^{\wedge} \Delta \phi_{3}^{\wedge} \ldots \Delta \psi_{N_{S^{-2}}}^{\wedge} \Delta \psi_{N_{S^{-1}}}^{\wedge}\right]^{T}
$$

\section{THE PROPOSED SYSTEM SIMULATION RESULTS AND DISCUSSION}

Now, show the simulation outcomes for suggested TSFE model along, Rayleigh fading hesitancy selective channel to SISO-OFDM system. Offer influences from Doppler frequency $f_{\mathrm{d}}$ (out coming because of proportional movement from nodes), the expansion agent, and the numeral from tracks on BER execution for suggested TSFE model. Investigating the influence of expansion agent $\Gamma$ on variety from suggested planner for various channel commands and Doppler frequencies. The simulation system, from a four-point DQPSK modulation on a RFC for bandwidth $B=108 \mathrm{~Hz}$, that matches with token interval $T \mathrm{~s}=10^{-7} \mathrm{~s}$. Knowing utilize a united energy lateness profile for $\mathrm{P}$ separate as well identically distributed tracks for average 0 as well contrast $\sigma^{2}{ }_{\mathrm{h}}=1 / P$. Lateness for kth track be $k T \mathrm{~s}$, wherever $\mathrm{k}=0,1 \ldots \mathrm{P}-1$. Effect for expansion coefficient $\Gamma$ over BER execution for TSFE mode be dual therefore achieved higher variety and improve the power every bit to noise proportion [27]. Figure 3 portrays effect of propagation over BER execution beneath optimal interleaving. Look status wherever Ns equal 1,024, $f_{\mathrm{d}}$ equal zero hertz and eighty hertz It determines $\mathrm{P}$ equal eight and assess BER execution for $\Gamma$ equal four, eight, and sixteen. Figure 3 illustrates TSFE mode suffers over variety wastage while $\Gamma$ equal four $(\Gamma<\mathrm{P})$ in every from $f_{\mathrm{d}}$ equal zero hertz and $f_{\mathrm{d}}$ equal eighty hertz. Propagation coefficient for $\Gamma$ equals four does not attain total variety while $\mathrm{P}$ equal eight at $f_{\mathrm{d}}$ equal zero hertz. Figure 3 so equal eight and sixteen at $f_{\mathrm{d}}$ equal zero hertz and eighty hertz.

Figure 4 offers effect $P$ on BER performance of suggested style. OFDM receiver could realize big hesitancy variety for P [28]. In Figure 4, studying effect of numeral of routes on BER performance, with regard to $\mathrm{P}$ equal four, $\mathrm{P}$ equal eight, for Ns equal five hundred twelve and $\Gamma$ equal eight, beneath 
optimum interleaving. Figure 4 appears, the suggested TSFE style attains total frequency variety if $\Gamma$ equal $\mathrm{P}$ with regard to $f_{\mathrm{d}}$ equal zero herts and $f_{\mathrm{d}}$ equal eighty hertz. The TSFE style attains variety arrange 4 for $\mathrm{P}$ equal four for every $\Gamma$ equal four and $\Gamma$ equal eight beneath $f_{\mathrm{d}}$ equal zero. So $\Gamma$ Greater or equal $\mathrm{P}$ does rise variety in with little proportional mobility.

Offer the Bit Error Rate implementation against Eb/NO for TSFE style for a half-ratio LDPC (six hundred forty-eight, three hundred twenty-four) encoder on a Rayleigh fading channel for $\mathrm{P}=4$. Utilize expansion agent of $\Gamma=16$ for optimum interleaving to propagation OFDM regime for Ns equal 1,296. Comparison inBER implementations for encoded as well coded (LDPC) for $f_{\mathrm{d}}$ equal eighty $\mathrm{Hz}$ as well one hundred eighty $\mathrm{Hz}$ to check effect of channel encrypting. Obvious of Figure 5 that LDPC encoder supplies important performance earning as compared to encoded DE for TSFE style. As showed in Figure 5 TSFE style for big propagation agent $\Gamma$ be very efficient in a weak SNR system. Moreover, the suggested TSFE method is very efficient beneath restricted transmission power and high proportional motion.

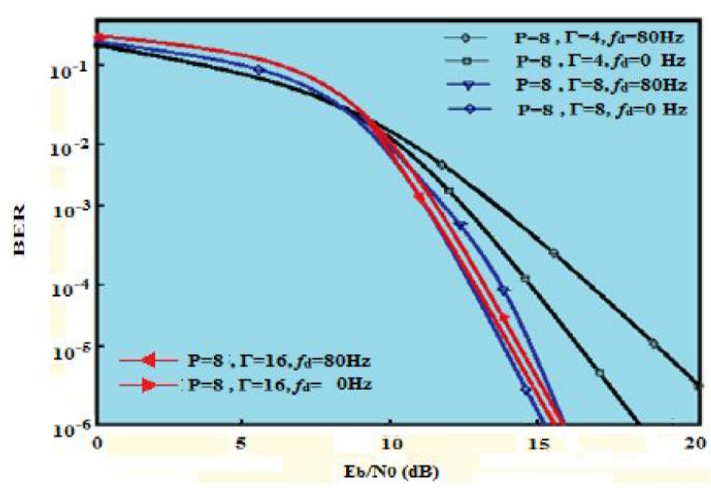

Figure 3. Impact for propagation coefficient $\Gamma$ over variety

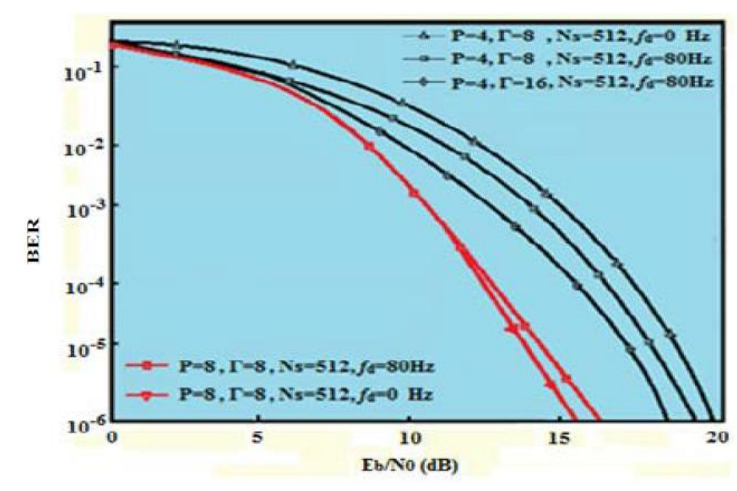

Figure 4. BER performance of TSFE method of single input single output OFDM regime

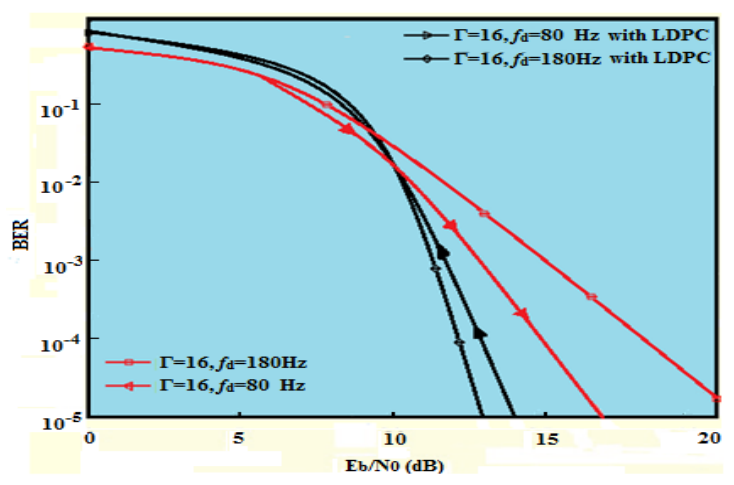

Figure 5. Bit error rate improvement in TSFE style for LDPC

\section{CONCLUSION}

The non-coherent SISO-OFDM communication regime was investigated in this research and modifications were implemented aiming at reducing SNR and enhancing relative mobility. It was suggested that TSFE to use DE at each frequency remoteness and propagation to avail frequency and time variety of the dynamic nodes. The simulation shows that DE along subcarriers attain higher varieties causing better ratio of power bit relative to noise. The technique used to achieve this outcome is the recurrence for input tokens along the remoteness time. In addition, LDPC FEC code was to improve the error rate. The results have shown that the scalability has been elevated in the field of decoding the information code. 


\section{ACKNOWLEDGEMENTS}

The authors would like to thank Mustansiriyah University (www.uomustansiriyah.edu.iq) Baghdad Iraq for its support in the present work.

\section{REFERENCES}

[1] N. Arne B. Svensson, "On Differentially Encoded Star 16 QAM with Differential Detection and Diversity," IEEE Transactions on Vehicular Technology, vol. 44, no. 3, 2011.

[2] L.H.-J. Lampe and R.F.H., "Fischer Comparison and Optimization of Differentially Encoded Transmission on Fading Channels," In 3rd Int. Symp. On Power-Line Communications, vol. 3, no. 6, 2013.

[3] Vahid Tarokh, "A Differential Detection Scheme for Transmit Diversity," IEEE Journal on Selected Areas in Communications, vol. 18, no. 7, 2000.

[4] D. Tse and P. Viswanath, Fundamentals of Wireless Communication, Cambridge, England: Cambridge University Press, 2007.

[5] S. M. Alamouti, "A Simple Transmitter Diversity Scheme for Wireless Communications," IEEE J. Select. Areas Commun., vol. 16, no. 4, 2015.

[6] Qi Zhang, Qian Yong, Jiayin Qin and A. Nallanathan, "On the Feasibility of the CJ Three-User Interference Aligment Scheme for SISO OFDM Systems," IEEE Communications Letters, vol. 18, no. 2, 2014.

[7] Qingjiang Shi, Weiqiang Xu, Dapeng Li, Yaming Wang, Xiaowei Gu, Wenshu Li, "On the Energy Efficiency Optimality of OFDMA for SISO-OFDM Downlink System," IEEE Communications Letters, vol. 17, no. 3, Mar 2013.

[8] C. Berrou and A. Glavieux, "Near Optimum Error Correcting Coding and DecodingTurbo-Codes," IEEE Trans. Commun., vol. 44, no. 10, 2011.

[9] Z. Wang, Z. Cui, and J. Sha, "VLSI Design for Low Density Parity-Check Code Decoding," Circuits and Systems Magazine, vol. 11, no. 1, 2011.

[10] Z. Wang, Y. Chen and K. K. Parhi, "Area Efficient Encoding of Quasi-Cyclic Low Density Parity Check Codes," In Proc. 2004 Int. Conf. on Acoustics, Speech, and Signal Processing (ICASSP'04), 2004.

[11] M. Zia, et al., "Bandwidth-Efficient Selective Retransmission for MIMO-OFDM Systems," Etri Journal, vol. 37, no. $1,2015$.

[12] A. Ramesh, A. Chockalingam and L.B. Milstein, "Performance of Noncoherent Turbo Detection on Rayleigh Fading Channels," IEEE Global Telecommun. Conf., - San. Antonio, TX, USA, vol. 2, pp.25-29, Nov. 2001.

[13] Xiaoli Ma, Georgios B. Giannakis, Bing Lu., "Block Differential Encoding for Rapidly Fading Channels," IEEE Transactions on Communication, vol. 52, no. 3, 2004.

[14] T. Petermann, S. Vogeler, K-D. Kammeyer, "Blind Turbo Channel Estimation in OFDM Receivers," In Proc. 35th Asilomar Conference on Signals, Systems and Computers, vol. 2, no. 3, 2001.

[15] H. Tatsunami, K. Ishibashi and H. Ochiai, "On the Performance of LDPC Codes with Differential Detection over Rayleigh Fading Channels," IEEE Veh. Technol. Conf., Melbourne, Australia, May 2006.

[16] E. Mo, P.Y. Kam, M.A. Armand, "LLR Metrics for LDPC Codes wit Quadrature Differential PSK Transmission, and their Performances," IEEE Int. Symp. Inf. Theory its Appl., Auckland, New Zealand, Dec. 2008.

[17] Zhang Y., Zhang C., Yan Z., Chen S. and Jiang H., "High Through Put Multi-Rate LDPC Decoder for Error Correction of Solid-State Drives," Proc. Of IEEE Workshop on Signal Processing Systems (SiPS), Hangzhou, China, Oct 2015.

[18] Khalid H. Sayhood and Wu Le Nan, "Differential Amplitude Phase Shift Keying (DAPSK) A New Modulation Method for a Turbo Code in Digital Radio Broadcasting," Engineering Journal of the University of Qatar, vol. 16, no. $5,2003$.

[19] M. Zia, et al., "Selective HARQ Transceiver Design for OFDM System," IEEE Communications Letters, vol. 17, no. $12,2013$.

[20] V. Tarokh and H. Jafarkhani, "A Differential Detection Scheme for Transmit Diversity," IEEE Journal on Selected Areas in Communications, vol. 18, no. 3, 2010.

[21] Elisa Mo., "LLR Metrics for LDPC Codes with Quadrature Differential PSK Transmission, and their Performances," 2008 International Symposium on Information Theory and Its Applications, Dec. 2008.

[22] S. Poorkasmaei and H. Jafarkhani, "Asynchronous Orthogonal Differential Modulation for Multiple Access Channels," IEEE Trans. Wireless Commun., vol. 3, no. 6, 2014.

[23] J. Proakis and M. Salehi, Digital Communications, NY,USA : McGraw-Hill, 2007.

[24] Z. Liu, Y. Xin and G.B. Giannakis, "Linear Constellation Precoding for OFDM with Maximum Multipath Diversity and Coding Gains," IEEE Trans. Commun., vol. 51, no. 3, 2003.

[25] X. Zhu and J. Xue, "On the Correlation of Subcarriers in Grouped Linear Constellation Precoding OFDM Systems over Frequency Selective Fading," IEEE Veh. Technol. Conf., Melbourne, Australia, vol. 3, no. 5. 2006.

[26] X. Wang, et al., "Efficient Non-pilot-aided Channel Length Estimation for Digital Broadcasting Receivers," IEEE Trans. Broadcast., vol. 55, no. 3, 2009.

[27] V.T. Nam, P.Y. Kam and Y. Xin, "LDPC Codes with BDPSK and Differential Detection over Flat Rayleigh Fading Channels," IEEE Conf. Global Telecommun., Washington, DC, USA, Nov. 2007.

[28] Asma Latif, Nasir D. Gohar, "BER Performance Evaluation and PSD Analysis of Non-Coherent Hybrid MQAMLFSK OFDM Transmission System," IEEE--ICET 2006 2nd International Conference on Emerging Technologies Peshawar, Pakistan, Nov. 2006. 


\section{BIOGRAPHIES OF AUTHORS}

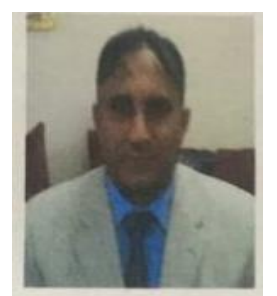

Karem J. Thajeel was born in Baghdad /Iraq 1959. He received B.Sc. degree in electrical \& Electronic engineering from Sarajevo/Bosnia 1984 and M.Sc. degree in Electronic \& communication engineering from University of Belgrade/Serbia 1986, in 1992. He had worked at airplanes maintenance factory in electronic department/Mosel/Iraq, in 1997 he had worked as a technical in electronic systems, he working now a lecturer in department of machinery and equipment technologies institute of technology middle technical university.

E-mail: kareemjabbar847@yahoo.com

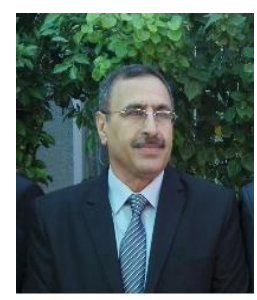

Izz K. Abboud was born in Erbil, Iraq 1962. He received B.Sc. degree in electrical \& Electronic engineering from Sarajevo, Bosnia 1984 and M.Sc. degree in Electronic \& communication engineering from University of Belgrade, Serbia 1986, in 1994. he had worked at airplanes repairing factory in electronic department, Baghdad, Iraq, in 1996 he had worked in Air force training institute communication department/ Baghdad, Iraq, in 2000 he worked as a lecturer in Institute of Technology/Baghdad/Iraq, in 2005 he worked as a lecturer in Al-Mustansiriyah University, Faculty of Engineering, Computer Engineering department, Baghdad, Iraq and currently he still three. His research interests electronics fields and communications fields, he has many research papers more than twenty in different journals and conferences.

E-mail: izz_kadhum@uomustansiriyah.edu.iq

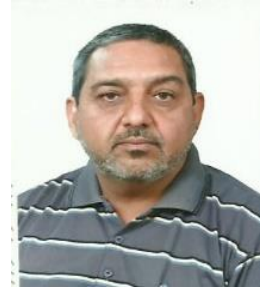

Laith A. Kunbar. He received B.Sc. in electrical engineering from Technological University, Baghdad, Iraq in 1981, M.Sc. in electronics and communications from Technological University, Baghdad, Iraq in 1990 and Ph.D. in electronics and communications from Technological University, Baghdad, Iraq in 2005. He had 30 years working and academic experiences in many factories and universities in Iraq. Currently, he is working as Assistant professor in AlMustansiriyah University / Faculty of Engineering /Computer Engineering Department, Iraq. His research interests include digital communications, Analogue and digital electronics and digital signal processing.

E-mail: laithkunbar@yahoo.com 